\title{
COMMUNICABLE DISEASES REPORT, NEW SOUTH WALES, FOR JULY AND AUGUST 2006
}

For updated information, including data and facts on specific diseases, visit www.health.nsw.gov.au and click on Infectious Diseases.

\section{TRENDS}

Tables 1 and 2 show reports of communicable diseases received for July and August 2006 in NSW; we are pleased to report that no measles cases were reported during this period. Figure 1 shows reports of selected communicable diseases, by month of onset, from January 2001 to August 2006.

\section{LYMPHOGRANULOMA VENEREUM EMERGES IN NSW}

Four cases of lymphogranuloma venereum (LGV) were notified to NSW Health in June 2006. The cases were identified after a batch of rectal swabs was tested using newly developed PCR and gene sequencing tests at St Vincent's Hospital. All four cases were homosexually active men who had presented with proctitis between October 2004 and March 2006 and initially tested positive for chlamydia. Public health units initiated an investigation into each case, but no direct links among the cases could be identified. These cases are the first cases of LGV notified in NSW. They follow an outbreak of LGV among gay men that was first identified in the Netherlands in late $2003^{1}$ and that has subsequently spread to other European countries and North America. ${ }^{2}$

LGV can cause proctitis in homosexually active men who are thought to acquire the infection following unprotected anal intercourse. It is caused by an especially aggressive form of chlamydia that invades lymph nodes and destroys the surrounding tissues. Symptoms usually develop about two months following infection and include an anal discharge, diarrhoea or constipation, abdominal pain and fever. ${ }^{3}$ Other sexually transmitted infections including HIV and hepatitis $\mathrm{C}$ have been associated with LGV infection. ${ }^{4}$

In October 2005 the Sexually Transmitted Infections in Gay Men Action Group (STIGMA) wrote to doctors in inner Sydney who care for gay men with information about LGV and how the infection can be diagnosed and treated.

In July 2006, NSW Health convened an expert panel to review the public health implications of LGV in NSW, and further recommendations will follow. The risk of LGV can be reduced through practicing safe sex. Homosexually active men should talk to their doctor about having regular sexual health checks, especially if they or a partner have symptoms. Specific intervention for infected patients include antibiotic therapy to both cure the infection and prevent further transmission, and partner notification and assessment.

\section{References}

1. Spaargaren J, Fennema HSA, Morré SA, de Vries HJC, Coutinho RA. New lymphogranuloma venereum Chlamydia trachomatis variant, Amsterdam. Emerg Infect Dis 2005; 11: 1090-2.

2. Blank S, Schillinger JA, Harbatkin D. Lymphogranuloma venereum in the industrialised world. Lancet North Am Ed 2005; 365: 1607-8.

3. Williams D, Churchill D. Ulcerative proctitis in men who have sex with men: an emerging outbreak. BMJ 2006; 332: 99-100.

4. Van der Bij AK, Spaargaren J, Morré SA, et al. Diagnostic and clinical implications of anorectal lymphogranuloma venereum in men who have sex with men: A retrospective case-control study. Clin Infect Dis 2006; 42: 186-94.

\section{MENINGOCOCCAL DISEASE}

Winter and spring are the peak periods for meningococcal disease in NSW. Until recently 200 to 250 cases of the disease were notified annually in the state. Of these, about half were due to serogroup B meningococcus and about a third were due to serogroup $\mathrm{C}$ meningococcus. However, the introduction of a vaccine against meningococcus $\mathrm{C}$ and a nationwide school-based immunisation program in 2003 and 2004 has led to a dramatic decline in serogroup C disease.

In 2005, 136 cases of meningococcal disease were reported in NSW, of which 73 (54\%) were due to serogroup B and $15(11 \%)$ to serogroup $\mathrm{C}$ meningococcus. To the end of August 2006, 68 cases had been reported, including $37(54 \%)$ due to meningococcus B and 7 (10\%) due to meningococcus $\mathrm{C}$.

For further information, see http://www.health.nsw.gov. $\mathrm{au} /$ infect/diseases.html.

\section{ENTERIC DISEASES}

Several viral gastroenteritis outbreaks in institutions were reported throughout July 2006 (Figure 1). In April 2006 an increase in the number of people attending emergency departments with gastroenteritis was detected through NSW Health's Public Health Real-time Emergency Department Surveillance System (PHREDSS). An increased number of notifications of outbreaks of viral gastroenteritis in institutional settings were also received in April, and outbreaks have been commonly reported since. By July, 216 outbreaks affecting more than 5000 people had been reported in 2006 across NSW, compared with 30 outbreaks affecting 300 people in the same period in 2005. The 2006 outbreaks were mainly reported from aged care facilities (141), hospitals (41) and childcare centres (30). The cause of the outbreaks was not confirmed for $74 \%$, but epidemiologically these outbreaks were typical of a viral infection. Norovirus was confirmed as the cause in $23 \%$ of outbreaks and rotavirus in $2 \%$. 
Norovirus is a common cause of vomiting and diarrhoea, especially in the winter months. Norovirus is very contagious and is spread easily between people. Rotavirus is the most common cause of viral gastroenteritis in children. Viral gastroenteritis is prevented by thorough hand washing with soap and water after using the toilet, and before preparing food or eating and drinking. For more information, see the Fact Sheet at: http://www.health.nsw. gov.au/infect/pdf/viral_gastro.pdf. Guidelines have been developed for the management of outbreaks in institutional settings (see: http://www.health.nsw.gov.au/pubs/2004/ gastroctrl_fs.html).

\section{A CLUSTER OF HEPATITIS A IN TRAVELLERS TO FIJI}

In August, Sydney South West Public Health Unit (SSWPHU) was notified of a person (Case 1) who had been diagnosed with hepatitis A. Approximately four weeks earlier, Case 1 had travelled to Fiji to attend a meeting, and after further investigation it was considered that Fiji was the likely source of the infection. Fifteen other Australians attended the meeting.

Hepatitis A is a viral infection of the liver. Symptoms include feeling unwell, aches and pains, fever, nausea, lack of appetite, abdominal discomfort and darkening of the urine, which is followed in a few days by jaundice. In some instances no symptoms are experienced at all. The incubation period is usually four weeks but can range from two to seven weeks. A positive serological test for hepatitis $\mathrm{A}$ is required to confirm the diagnosis. Passive immunisation with normal human immunoglobulin (NHIG), which contains antibodies derived from the pooled plasma of blood donors, is effective in preventing hepatitis A if given within two weeks of the person being exposed to the virus.

Soon after Case 1 had been notified to SSWPHU, a further case of hepatitis A in another person who attended the same meeting was notified. Public health unit staff obtained a list of names and contact details of participants from the organiser of the meeting. Attempts were made to contact each of the attendees by telephone to ascertain if they had become unwell with symptoms of hepatitis A. Contact was made with 10 (out of 14) participants by telephone, and it was discovered that hepatitis $\mathrm{A}$ had been diagnosed in a third participant, a resident of Queensland. The remaining nine participants who were contacted denied any symptoms of hepatitis A. Four of the meeting participants had received hepatitis A vaccine prior to travelling to Fiji, and one reported immunity to hepatitis A due to previous illness. An information letter was mailed or sent electronically to all participants advising them of their risk of hepatitis A infection and the symptoms of the illness.

Further follow-up of all meeting participants was attempted four weeks after the initial notification. Contact was made with seven of the 13 participants who were not cases. All these people reported that they had remained well; however, five attended their general practitioner (GP) to obtain further advice on this issue. One person received hepatitis A vaccination from the GP following the trip, but this was the second dose of vaccine of a course commenced prior to travelling.

The cluster of three cases of hepatitis A in travellers to Fiji highlights the importance of hepatitis A vaccination prior to travel to endemic countries. It is important to educate both travellers and general practitioners about the value of individually targeted travel advice (such as safe eating and drinking) and vaccinations prior to travelling to developing countries. The meeting organisers have also been advised to recommend hepatitis A vaccination in future courses.

\section{HEPATITIS A PROPHYLAXIS CLINIC}

In August, a case of hepatitis A was reported to Sydney South West Public Health Unit (SSWPHU). Follow-up revealed that the case worked at a school tuckshop on 11 August whilst infectious, preparing ready-to-eat foods. Although the case did not experience any vomiting or diarrhoea while at the tuckshop, a risk assessment by the public health unit raised concerns about opportunities for transmission during the preparation of food.

A decision was made to offer NHIG to any member of the school community who may have consumed readyto-eat foods at the tuckshop on 11 August. On Tuesday 22 August the school provided a letter to parents and students including fact sheets about hepatitis A and NHIG, and sought consent for students at risk to be passively immunised. Two clinics, with staff from Sydney West, South Eastern Sydney Illawarra, Sydney South West Area Health Services, and NSW Department of Health, were held at the school on August 23 and 24. Information and NIGH were given to 568 students, teachers and tuckshop workers. The NSW Food Authority inspected the tuckshop and provided information regarding safe food handling.

No secondary cases of hepatitis A have been identified in relation to this incident but heightened surveillance will continue until early October.

Good food safety practices are crucial in all food settings, including smaller operations staffed by volunteers such as a tuckshop. 공 
FIGURE 1

\section{REPORTS OF SELECTED COMMUNICABLE DISEASES, NSW, JAN 2001 TO AUGUST 2006, BY MONTH OF ONSET}

Preliminary data: case counts in recent months may increase because of reporting delays.

Laboratory-confirmed cases only, except for measles, meningococcal disease and pertussis $\mathrm{BFV}=$ Barmah Forest virus infections

Lab conf $=$ laboratory confirmed
RRV = Ross River virus infections
Men $\mathrm{Gp} \mathrm{C}$ and $\mathrm{Gp} \mathrm{B}=$ meningococcal disease due to serogroup $C$ and serogroup $B$ infection, other/unk = other or unknown serogroups.

NB: multiple series in graphs are stacked, except gastroenteritis outbreaks.

NB: Outbreaks are more likely to be reported by nursing homes and hospitals than by other institutions

\begin{tabular}{|rr}
\hline \multicolumn{2}{|l|}{ NSW population } \\
Male & $50 \%$ \\
$<5$ yrs & $7 \%$ \\
$5-24$ yrs & $27 \%$ \\
$25-64$ yrs & $53 \%$ \\
$65+$ yrs & $13 \%$ \\
Rural & $46 \%$
\end{tabular}

Arbovirus

\begin{tabular}{|rc|}
\hline \multicolumn{3}{|c|}{ Jun 06-Aug 06 } \\
Male & $51 \%$ \\
$<5$ & $40 \%$ \\
$5-24$ & $25 \%$ \\
$25-64$ & $33 \%$ \\
$65+$ & $2 \%$ \\
Rural & $42 \%$ \\
\hline
\end{tabular}

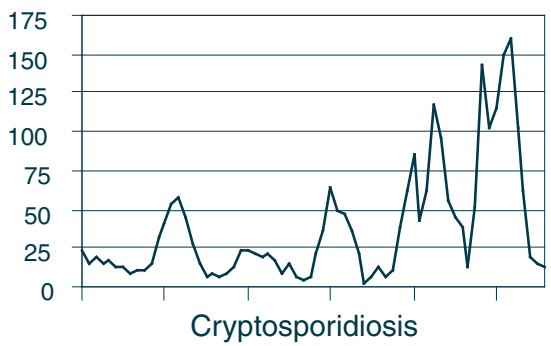

Jun 06-Aug 06

Male $87 \%$

$<5$
-5

5-24 31\%

$25-64 \quad 68 \%$

Rural $17 \%$

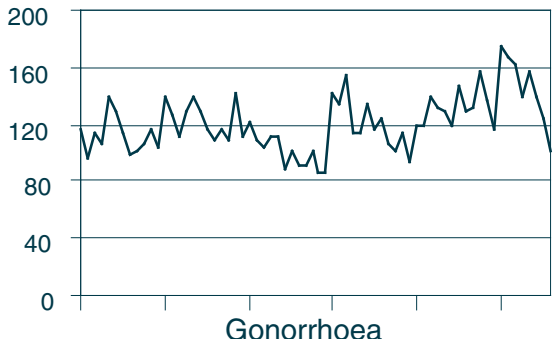

\begin{tabular}{|cc|}
\hline \multicolumn{2}{|c|}{ Jun 06-Aug 06 } \\
Male & $40 \%$ \\
$<5$ & $0 \%$ \\
$5-24$ & $32 \%$ \\
$25-64$ & $56 \%$ \\
$65+$ & $12 \%$ \\
Rural & $12 \%$ \\
\hline
\end{tabular}

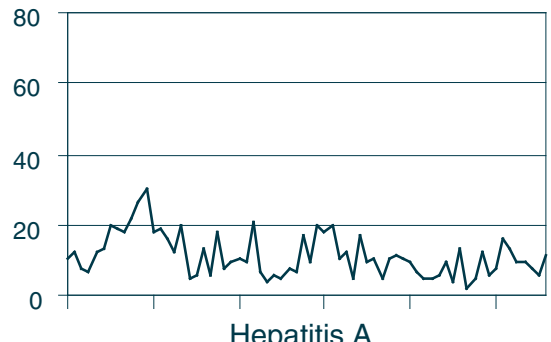

Hepatitis A

\begin{tabular}{|lr|}
\hline \multicolumn{2}{|c|}{ Jun 06-Aug 06} \\
All outbreaks & 122 \\
Nursing homes & 85 \\
Hospitals & 25 \\
Child care & 10 \\
Schools & 0 \\
Other & 2 \\
\hline
\end{tabular}

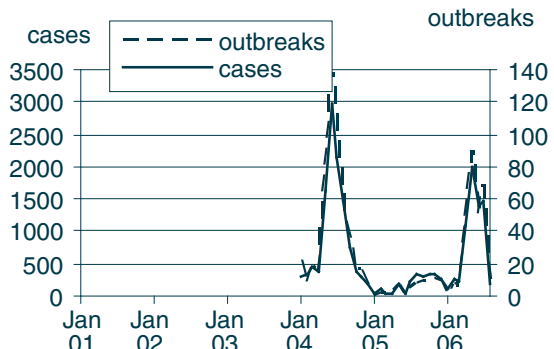

Gastroenteritis outbreaks in institutions
Jun 06-Aug 06 Male $43 \%$

$<5 \quad 0 \%$ $5-24 \quad 0 \%$ 25-64 57\%

$65+\quad 43 \%$
Rural

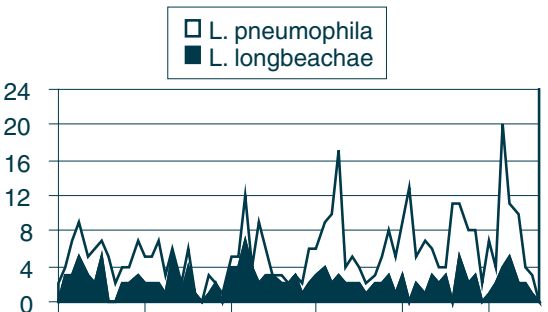

Legionellosis

Rural $29 \%$

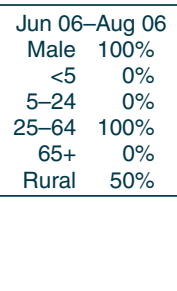

Measles

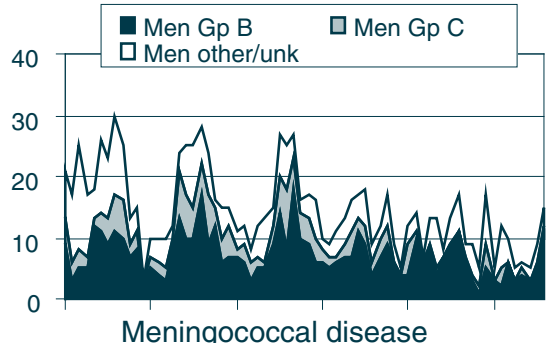

\begin{tabular}{|rc|}
\hline \multicolumn{2}{|c|}{ Jun 06-Aug 06 } \\
Male & $35 \%$ \\
$<5$ & $21 \%$ \\
$5-24$ & $48 \%$ \\
$25-64$ & $31 \%$ \\
$65+$ & $0 \%$ \\
Rural & $38 \%$ \\
\hline
\end{tabular}

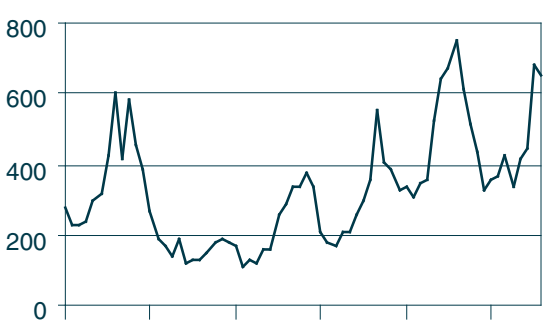

Pertussis
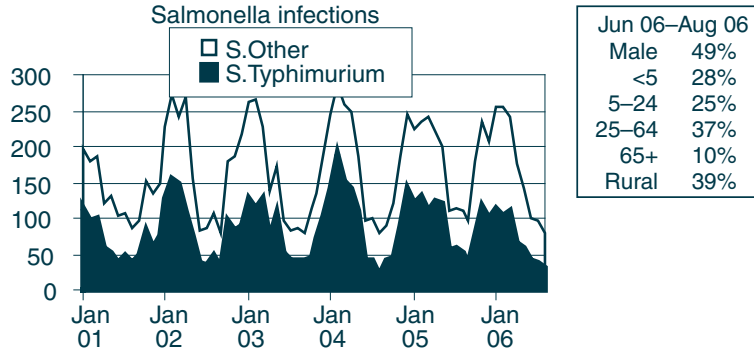

Salmonella infections

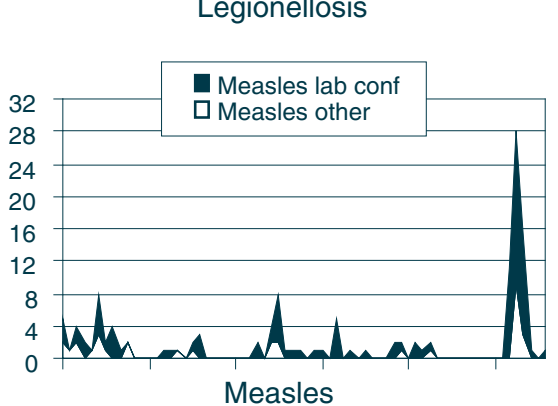




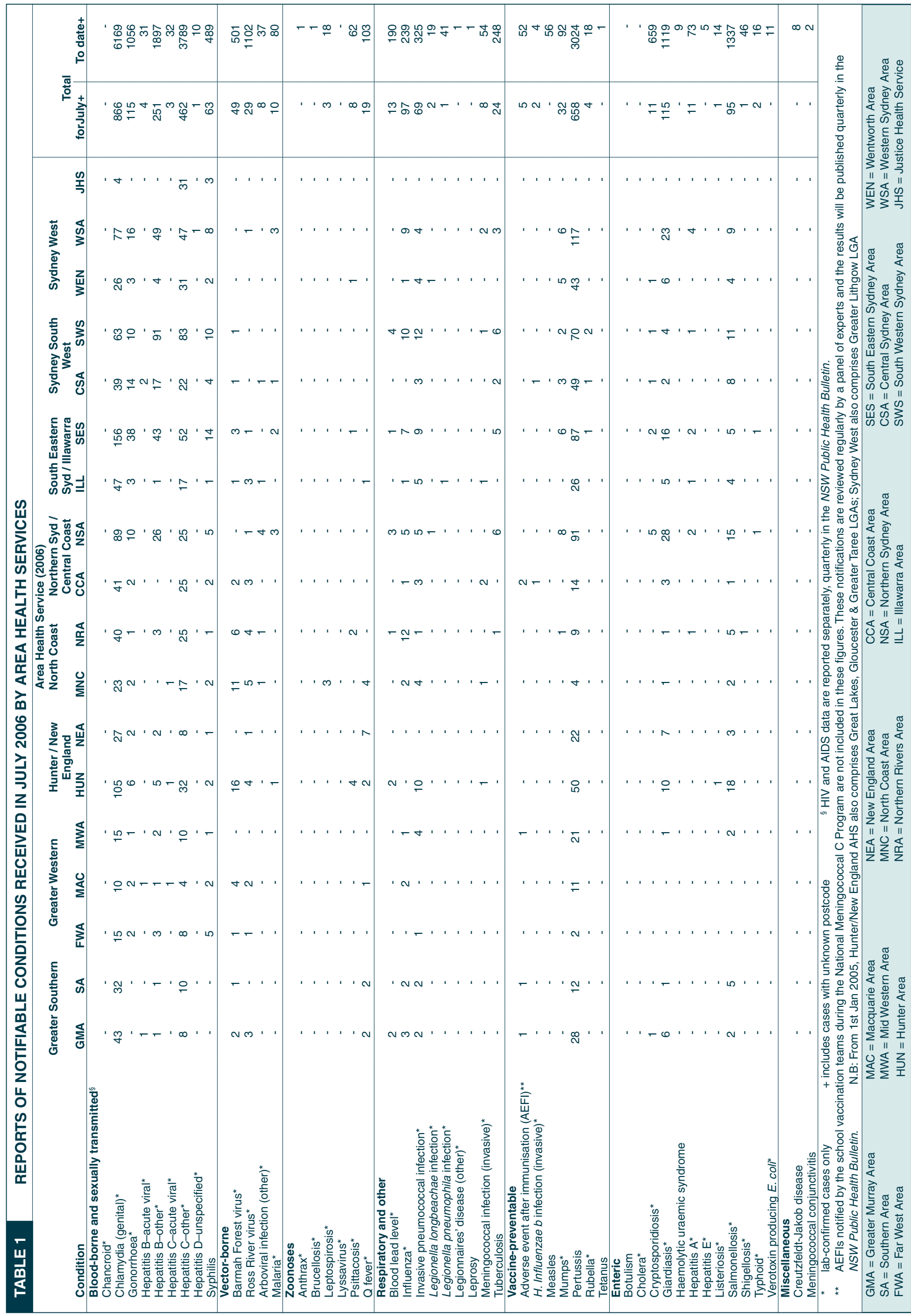




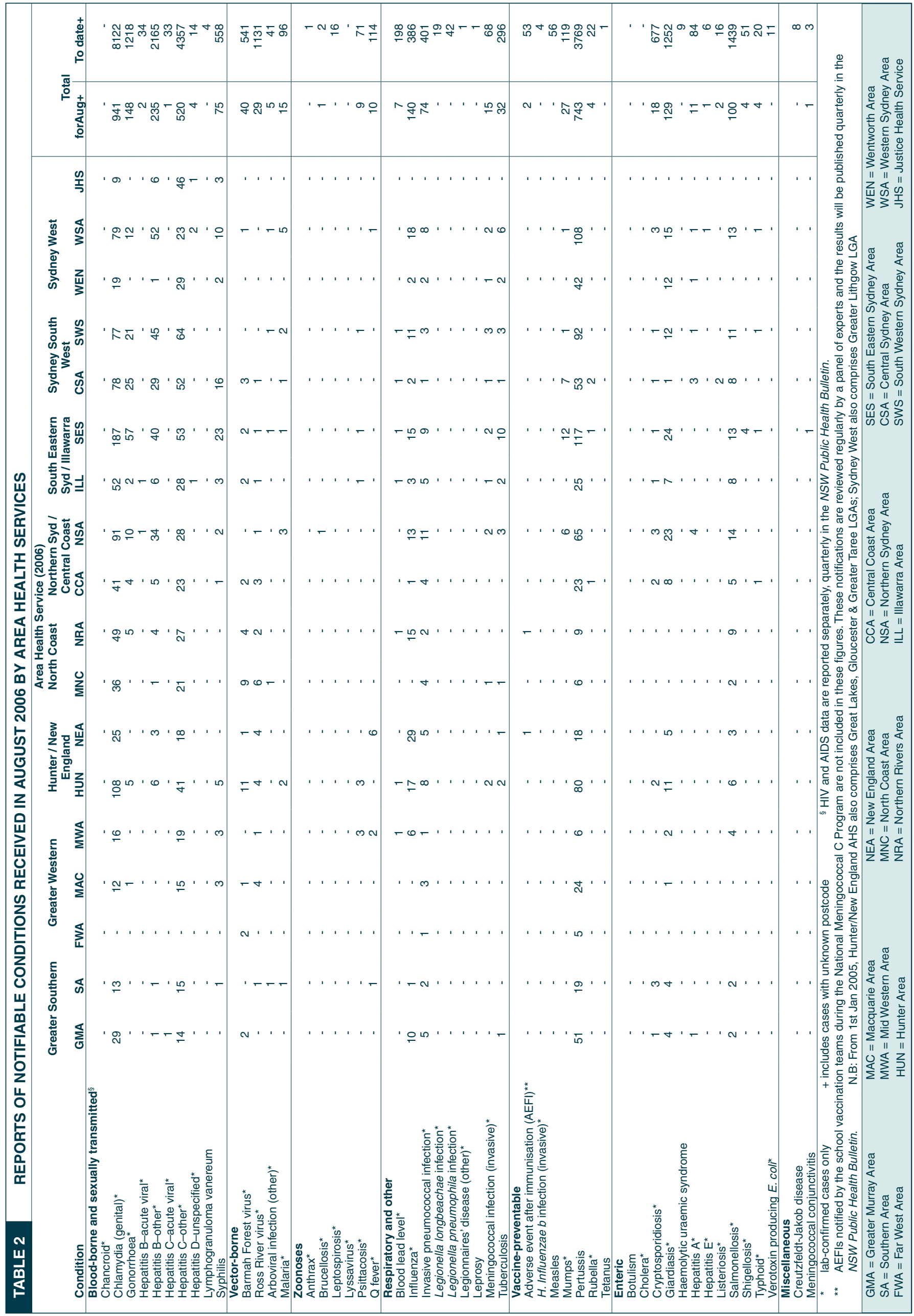

